\title{
Four-dimensional equations for the study of electromagnetic plasma turbulence in a drift kinetic limit
}

\author{
Evgeny A. Gorbunov ${ }^{1}$ and Bogdan Teaca ${ }^{1,2}$ \\ ${ }^{1}$ Coventry University, Coventry CV1 5FB, United Kingdom \\ ${ }^{2}$ University of Craiova, 13 A.I. Cuza Street, 200585 Craiova, Romania
}

\begin{abstract}
For a magnetised plasma in a straight magnetic guide field, we derive a set of four-dimensional kinetic equations, which can capture electromagnetic turbulence in the drift kinetic limit. To do so, we start from the gyrokinetic equations, employ a Laguerre decomposition in the perpendicular velocity direction, retain only the dominant gyroaverage contributions and only the first two Laguerre moments that source the electromagnetic fluctuations. The model conserves free energy, and can describe electromagnetic turbulence for a plasma at the transition between fluid and gyrokinetic regimes $\left(k_{\perp} \rho_{i} \approx 1\right.$ range of scales), as dominant finite-Larmor-radius (FLR) effects are considered. In addition to the three dimensions in positions space, we retain the parallel velocity dependence, which we describe via a Hermite representation. Employing this system, but without any other physics based assumptions for the plasma species that can bias results, will allow us to investigate how fluid effects transition into the kinetic range, and analyse the interplay between spatial and velocity space mixing for electromagnetic plasma turbulence.
\end{abstract}

\section{INTRODUCTION}

In astrophysical settings, the use of magnetohydrodynamics (MHD) is successful in the description of the large scale dynamics of plasma turbulence [e.g. 4, 7, 12, 38, 40, 41. At the same time, the use of kinetic formalisms allows us to probe micro-scales in the collisionless limit [e.g. 6, 13, 27, 37]. Gyrokinetic (GK) theory in particular is used for the study of turbulence in collisionless magnetised plasma at sub-gyroradius scales [15] 17, 23, 28, 29, 33, 35]. Theoretical considerations [24, 25], numerical simulations [28, 35] and satellite observations [2, 3] have shown that at the ion Larmor (gyroradius) scale, as turbulence transitions from a fluid to a kinetic dynamical regime, a break occurs in the spectra of various quantities. While the scientific community is aware of this spectral break for the last decade, recent observations from the Parker Solar Probe [e.g. 9, 10, 36, 39, have spurred an interest for understanding the dynamics of this transition.

To study this transition in detail, while accounting for the collisionless regime, a GK formalism can be used for magnetised plasma. Even when the strength of the magnetic guide fields is not that large and the GK ordering is weakened, such as in the solar wind, GK theory is still useful in capturing the main dynamical properties, such as the kinetic Alfvén waves (KAW) cascade, Landau damping, linear and nonlinear phase space mixing. While the five-dimensional phase space for GK represents a simplification over the full Maxwell-Vlasov system, it is still too cumbersome to use for systematic studies of the fluid-kinetic transition. For this reason, starting from the electromagnetic GK equations, we will derive a four-dimensional drift kinetic system that accounts for the dominant finite Larmor radius (FLR) effects. We preserve the three spatial directions and the parallel velocity direction that will allow us to account for the important parallel phase mixing dynamics.

In essence, starting from the GK equations and employing a Laguerre decomposition in the perpendicular velocity direction (\$II), retaining only the dominant gyroaverage contributions and only the first two Laguerre moments for the distribution functions, we obtain a closed set of four-dimensional kinetic equations valid in the large-scale drift kinetic limit (\$III). In addition to the three-dimensions in positions space, we retain the parallel velocity direction, which we describe via a Hermite representation ( $(\mathrm{IV})$. The model we will propose conserves free energy and describes electromagnetic turbulence for a plasma at the transition between fluid and gyrokinetic regimes $\left(k_{\perp} \rho_{i} \approx 1\right.$ range of scales), accounting for FLR effects. It allows for Landau damping, and can allow or potentially prohibit parallel mixing to occur due to turbulent plasma echos [18, 21, 26]. The strength of the model consists in linking with GK at $k_{\perp} \rho_{i}>1$ scales, while allowing for any (gyro) fluid nature for the ions or electrons species to emerge naturally and without any other physical assumption. Numerically, it will represent a good compromise that will allow for the study of how fluid constraints (similar to magnetic-helicity and cross-helicity in MHD) can impact the transition towards the kinetic dynamics, or how the microphysics affect the macrophysics of the plasma 22. Considering that the nonlinear interaction of spatial structures and the velocity phase mixing in the parallel direction represent the main dynamics of kinetic turbulence, the model also offers a simplified system for the study of the interplay between spatial turbulence and parallel phase mixing when electromagnetic interactions are present. 


\section{GK EQUATIONS IN LAGUERRE SPACE AS A STARTING POINT}

\section{A. The gyrokinetic equations}

The starting point for our derivation is given by the gyrokinetic (GK) equations in the presence of a straight magnetic guide field (acting here in the $z$-direction, $B_{0} \hat{\mathbf{z}}$ ). The community regularly employs the GK equations in the study of kinetic plasma at sub-gyroradius scales $\left(k_{\perp} \rho_{i}>1\right)$, and we have used them to probe the fundamental nature of GK turbulence 29 33. A simple $\delta f$-derivation of the GK equations for a Maxwellian background distribution function $\left(F_{s}\right)$ is presented in 15, 25 and as such, we will not insist here on the GK construction.

For $h_{s}=h_{s}\left(\mathbf{R}_{s}, v_{\|}, \mu\right)$ the non-adiabatic part of the gyrokinetic distribution function (with the particle perturbed distribution function being found as $\delta f_{s}=-q_{s} \phi F_{s} / T_{s}+h_{s}$ ), we simply list below the GK equations in non-dimensional form and in the absence of collisions

$$
\frac{\partial h_{s}}{\partial t}+\frac{1}{B_{0}}\left\{\chi_{s}, h_{s}\right\}+v_{T_{s}} v_{\|} \frac{\partial h_{s}}{\partial z}=\frac{q_{s}}{T_{s}} F_{s} \frac{\partial \chi_{s}}{\partial t},
$$

where $\left\{\chi_{s}, h_{s}\right\}=\left(\nabla \chi_{s} \times \nabla h_{s}\right) \cdot \hat{\mathbf{z}}$ is the nonlinearity written in term of a Poisson bracket structure, $v_{T s}=\left(\sqrt{2 T_{s} / m_{s}}\right)$ is the thermal velocity for a species $s$ of charge $q_{s}$, mass $m_{s}$ and background temperature $T_{s}$ (all expressed in terms of reference units). The perpendicular velocity dependence is expressed in term of the magnetic moment $\mu$ (in non-dimensional form, $\mu B_{0}=v_{\perp}^{2}$ ) and the equilibrium distribution function $F_{s}$ has the simple form

$$
F_{s}=\pi^{-3 / 2} e^{-v_{\|}^{2}-\mu B_{0}} .
$$

Here, $\chi_{s}$ is the gyroaveraged gyrokinetic potential. It is convenient to represent it in Fourier space, as the gyroaverage operations reduce to the multiplication of Bessel functions of zero $\left(J_{0}\right)$ and first $\left(J_{1}\right)$ order,

$$
\chi_{s}\left(\mathbf{k}, \mu, v_{\|}\right)=\sum_{\mathbf{k}} e^{i \mathbf{k} \cdot \mathbf{R}_{s}}\left[J_{0}\left(\lambda_{s}\right)\left(\phi(\mathbf{k})-v_{T_{s}} v_{\|} A_{\|}(\mathbf{k})\right)+\frac{\mu T_{s}}{q_{s}} \frac{2 J_{1}\left(\lambda_{s}\right)}{\lambda_{s}} B_{\|}(\mathbf{k})\right] .
$$

The electrostatic potential $(\phi)$, magnetic potential in the parallel direction $\left(A_{\|}\right)$, and magnetic fluctuation in the parallel direction $\left(B_{\|}\right)$are obtained in wave space from their respective field equations,

$$
\begin{aligned}
\phi(\mathbf{k}, t) & =\pi B_{0} \sum_{s} q_{s} n_{s} \int_{-\infty}^{+\infty} d v_{\|} \int_{0}^{\infty} d \mu J_{0}\left(\lambda_{s}\right) h_{s}\left(\mathbf{k}, v_{\|}, \mu, t\right) / \sum_{s} \frac{q_{s}^{2} n_{s}}{T_{s}}, \\
A_{\|}(\mathbf{k}, t) & =\frac{\pi \beta}{2 k_{\perp}^{2}} B_{0} \sum_{s} q_{s} n_{s} v_{T_{s}} \int_{-\infty}^{+\infty} d v_{\|} v_{\|} \int_{0}^{\infty} d \mu J_{0}\left(\lambda_{s}\right) h_{s}\left(\mathbf{k}, v_{\|}, \mu, t\right), \\
B_{\|}(\mathbf{k}, t) & =-\frac{\pi \beta}{4} B_{0} \sum_{s} m_{s} n_{s} v_{T_{s}}^{2} \int_{-\infty}^{+\infty} d v_{\|} \int_{0}^{\infty} d \mu \mu \frac{2 J_{1}\left(\lambda_{s}\right)}{\lambda_{s}} h_{s}\left(\mathbf{k}, v_{\|}, \mu, t\right),
\end{aligned}
$$

where $\beta=8 \pi n_{\text {ref }} T_{\text {ref }} / B_{\text {ref }}^{2}$ is defined in term of the reference temperature $T_{\text {ref }}$, density $n_{\text {ref }}$ and magnetic intensity $B_{\text {ref. }}$. We also use

$$
\begin{aligned}
\lambda_{s} & =\sqrt{2 \mu B_{0} b_{s}}, \\
b_{s} & =\left(\frac{k_{\perp} v_{T s}}{\sqrt{2} \Omega_{s}}\right)^{2}=\frac{k_{\perp}^{2} \rho_{s}^{2}}{2} .
\end{aligned}
$$

From the GK equations above, we see that the gyroaverage operations, captured by the Bessel functions, smear $k_{\perp}$ structures into $\mu$ structures at $k_{\perp} \rho_{i}>1$. In the $k_{\perp} \rho_{i} \ll 1$ limit, for which the Bessel functions tend towards one, if no initial $\mu$ structure is present in $h_{s}$, the GK equations cannot generate subsequent $\mu$ structures and we can integrate over the $\mu$ direction without any loss of information. To see this better, and to integrate $\mu$ in a consistent way, we make use of a Laguerre transform approach.

\section{B. Laguerre transform}

A recent detailed approach for the use of a Laguerre transform for the GK system can be found in [20]. Here, we present only the information needed to understand the transformations we will employ in our derivation. A 
notable simplification is that we resume to a constant $B_{0}$ rather than the more general $B_{0}(z)$ approach employed by [20]. However, we do not resume to the simpler electrostatic limit, and consider electromagnetic fluctuations in our derivation.

For convenience, we define the Laguerre (basis) functions as

$$
\begin{aligned}
& \psi^{l}\left(\mu B_{0}\right)=(-1)^{l} e^{-\mu B_{0}} L_{l}\left(\mu B_{0}\right), \\
& \psi_{l}\left(\mu B_{0}\right)=(-1)^{l} L_{l}\left(\mu B_{0}\right),
\end{aligned}
$$

where $L_{l}(x)=\frac{e^{x}}{l !} \frac{d^{l}}{d x^{l}} x^{l} e^{-x}$ are Laguerre polynomials. The Laguerre functions obey the orthonormality condition,

$$
\int_{0}^{+\infty} d \mu B_{0} \psi^{k} \psi_{l}=\delta_{k l}
$$

which results from the orthogonality condition of the Laguerre polynomials $L_{l}(x)$ around the $e^{-x}$ weight function (note that $\psi_{l}(x) e^{-x}=\psi^{l}(x)$ ). In our derivation, we will also make use of the recurrence relation

$$
(l+1) \psi^{l+1}=\left(\mu B_{0}-2 l-1\right) \psi^{l}-l \psi^{l-1} .
$$

The decomposition of a function $f(\mu)$ in term of the Laguerre basis $\psi^{l}$ is done as

$$
f(\mu)=\sum_{l=0}^{\infty} \psi^{l}\left(\mu B_{0}\right) \widehat{f}_{l}
$$

with the spectral amplitude $\widehat{f}_{l}$ for the Laguerre modes $l$ being found simply as

$$
\widehat{f_{l}}=\int_{0}^{\infty} d \mu B_{0} \psi_{l} f(\mu) .
$$

The Laguerre transforms given by eqs. 13 14 allows us to obtain the GK equations in Laguerre space. Apart from projecting $h_{s}$, we will also need to project $J_{0}\left(\lambda_{s}\right)$ and $\frac{2}{\lambda_{s}} J_{1}\left(\lambda_{s}\right)$ onto the Laguerre basis. Note that $\chi_{s}$ depends on $\mu$ solely through the Bessel functions. In the drift kinetic limit, retaining the contribution of the Bessel functions will allow for finite Larmor radius (FLR) effects to be preserved [8].

Remembering that $\lambda_{s}=\sqrt{2 \mu B_{0} b_{s}}$, and omitting the species subscript $s$, we write the Bessel functions of order $n$ in term of the basis $\psi_{l}$, with the coefficients $\mathcal{J}_{n l}$ obtained via a generating function, i.e.

$$
J_{n}\left(\sqrt{2 \mu B_{0} b}\right)=\sum_{l} \psi_{l} \mathcal{J}_{n l}=\sum_{l} \psi_{l} \frac{b^{l}}{l !} \frac{\partial^{l}}{\partial b^{l}}\left\langle J_{n}\right\rangle
$$

where

$$
\left\langle J_{n}\right\rangle=\int_{0}^{\infty} e^{-\mu B_{0}} J_{n}\left(\sqrt{2 \mu B_{0} b}\right) B_{0} d \mu .
$$

Obtaining the same decomposition for the $\frac{2}{\lambda_{s}} J_{1}\left(\lambda_{s}\right)$ function requires an extra manipulation. Using the well-known recurrence formula for the Bessel functions, we arrive at the expression

$$
\frac{2}{\lambda_{s}} J_{1}\left(\lambda_{s}\right)=J_{0}\left(\lambda_{s}\right)+J_{2}\left(\lambda_{s}\right)=\sum_{l} \psi_{l} \tilde{\mathcal{J}}_{1 l}=\sum_{l} \psi_{l} \frac{b^{l}}{l !} \frac{\partial^{l}}{\partial b^{l}}\left(\left\langle J_{0}\right\rangle+\left\langle J_{2}\right\rangle\right) .
$$

The coefficients for the $\frac{2}{\lambda_{s}} J_{1}\left(\lambda_{s}\right)$ function are labeled as $\tilde{\mathcal{J}}_{1 l}$ (the tilde notation is used to distinguish it form the coefficients of $\left.J_{1}\left(\lambda_{s}\right)\right)$ and we express it via the generating function involving $\left\langle J_{0}\right\rangle$ and $\left\langle J_{2}\right\rangle$. From $(16)$ we obtain

$$
\begin{aligned}
& \left\langle J_{0}\right\rangle=e^{-b / 2}, \\
& \left\langle J_{2}\right\rangle=-\left(\frac{2}{b}+1\right) e^{-b / 2}+\frac{2}{b},
\end{aligned}
$$

and the coefficients for the Bessel functions of interest are obtained as

$$
\begin{aligned}
& \mathcal{J}_{0 l}=\frac{b^{l}}{l !} \frac{\partial^{l}}{\partial b^{l}} e^{-b / 2} \\
& \tilde{\mathcal{J}}_{1 l}=\frac{b^{l}}{l !} \frac{\partial^{l}}{\partial b^{l}}\left[\left(1-e^{-b / 2}\right) \frac{2}{b}\right] .
\end{aligned}
$$

Compared to 20, since we account for electromagnetic fluctuations, we needed to obtain $\tilde{\mathcal{J}}_{1 l}$. For clarity, the GK equation in Laguerre space are presented next. 


\section{Gyrokinetic system in Laguerre basis}

We start by looking at the field equations in Laguerre basis. For $\phi$, given by eq. (4), we use (13) for the representation of $h_{s}$ and 15 for the representation of $J_{0}\left(\lambda_{s}\right)$. We obtain

$$
\begin{aligned}
\phi(\mathbf{k}, t) & =\pi B_{0} \sum_{s} q_{s} n_{s} \int_{-\infty}^{+\infty} d v_{\|} \int_{0}^{\infty} d \mu \sum_{l} \psi_{l} \mathcal{J}_{0 l} \sum_{m} \psi^{m} \widehat{h}_{s m} / \sum_{s} \frac{q_{s}^{2} n_{s}}{T_{s}} \\
& =\pi \sum_{s} q_{s} n_{s} \int_{-\infty}^{+\infty} d v_{\|} \sum_{l} \mathcal{J}_{0 l} \widehat{h}_{s l} / \sum_{s} \frac{q_{s}^{2} n_{s}}{T_{s}}
\end{aligned}
$$

where we used the orthogonality condition (11) to obtain the second line. The same approach is used for $A_{\|}$, which gives

$$
A_{\|}(\mathbf{k}, t)=\frac{\pi \beta}{2 k_{\perp}^{2}} \sum_{s} q_{s} n_{s} v_{T_{s}} \int_{-\infty}^{+\infty} d v_{\|} v_{\|} \sum_{l} \mathcal{J}_{0 l} \widehat{h}_{s l}
$$

For the $B_{\|}$field equation, after the Laguerre representation for each contributing function is inserted in the first step, we extract $\mu \psi^{m}$ from the recurrence relation $\sqrt{12}$ in the second step, and use the orthogonality condition (11) in the last step, obtaining

$$
\begin{aligned}
B_{\|}(\mathbf{k}, t) & =-\frac{\pi \beta}{4} B_{0} \sum_{s} m_{s} n_{s} v_{T_{s}}^{2} \int_{-\infty}^{+\infty} d v_{\|} \int_{0}^{\infty} d \mu \mu \sum_{l} \psi_{l} \tilde{\mathcal{J}}_{1 l} \sum_{m} \psi^{m} \widehat{h}_{s m} \\
& =-\frac{\pi \beta}{4} \sum_{s} m_{s} n_{s} v_{T_{s}}^{2} \int_{-\infty}^{+\infty} d v_{\|} \int_{0}^{\infty} d \mu \sum_{l} \psi_{l} \mathcal{J}_{1, l} \sum_{m}\left[(2 m+1) \psi^{m}+\right. \\
& \left.m \psi^{m-1}+(m+1) \psi^{m-1}\right] \widehat{h}_{s m} \\
& =-\frac{\pi \beta}{2} \sum_{s} \frac{n_{s} T_{s}}{B_{0}} \int_{-\infty}^{+\infty} d v_{\|} \sum_{m} \mathcal{J}_{1, l}\left[(2 l+1) \widehat{h}_{s, l}+\widehat{h}_{s, l-1}+(l+1) \widehat{h}_{s, l+1}\right] .
\end{aligned}
$$

With the electromagnetic potentials and fields determined, to project the gyrokinetic potential (3) onto the Laguerre basis, we need to express the Bessel functions contributions and use again the recurrence relation (12) to express $\mu \psi_{m}$, before employing the orthogonality condition (11). The $\widehat{\chi}_{l}$ spectral amplitude gives

$$
\begin{aligned}
\widehat{\chi}_{l}(\mathbf{k})= & \int_{0}^{\infty} d \mu B_{0} \psi^{l}\left[\left(\phi(\mathbf{k})-v_{T_{s}} v_{\|} A_{\|}(\mathbf{k})\right) \sum_{m} \psi_{m} J_{0 l}+\frac{\mu T_{s}}{q_{s}} B_{\|}(\mathbf{k}) \sum_{m} \psi_{m} \tilde{\mathcal{J}}_{1 l}\right] \\
= & B_{0} \int_{0}^{\infty} d \mu \psi^{l}\left(\phi(\mathbf{k})-v_{T_{s}} v_{\|} A_{\|}(\mathbf{k})\right) \sum_{m} \psi_{m} J_{0 m}+ \\
& \quad+\frac{T_{s}}{q_{s} B_{0}} B_{\|}(\mathbf{k}) \sum_{m}\left[(m+1) \psi_{m+1}+(2 m+1) \psi_{m}+m \psi_{m-1}\right] \tilde{\mathcal{J}}_{1 m} \tilde{\mathcal{J}}_{1 l} \\
= & \mathcal{J}_{0 l}\left(\phi(\mathbf{k})-v_{T_{s}} v_{\|} A_{\|}(\mathbf{k})\right)+\frac{T_{s}}{q_{s} B_{0}} B_{\|}(\mathbf{k})\left((l+1) \tilde{\mathcal{J}}_{1, l+1}+(2 l+1) \tilde{\mathcal{J}}_{1, l}+l \tilde{\mathcal{J}}_{1, l-1}\right) .
\end{aligned}
$$

To obtain the GK equation (1) on the Laguerre basis, we look at each term independently. We remind the reader that the $\mu$ dependence of the potential $\chi$ is due to the Bessel functions, and as such, the Laguerre expansion of $\chi$ occurs in term of the basis $\psi_{l}$. The linear terms are computed in a straightforward maner,

$$
\begin{aligned}
& \int_{0}^{\infty} d \mu B_{0} \psi_{l} \sum_{m} \psi^{m} \frac{\partial \widehat{h}_{m}}{\partial t}=\frac{\partial \widehat{h}_{l}}{\partial t}, \\
& \int_{0}^{\infty} d \mu B_{0} \psi_{l} \sum_{m} \psi^{m} v_{T_{s}} v_{\|} \frac{\partial \widehat{h}_{m}}{\partial z}=v_{T_{s}} v_{\|} \frac{\partial \widehat{h}_{l}}{\partial z}, \\
& \int_{0}^{\infty} d \mu B_{0} \frac{q_{s}}{T_{s}} \pi^{-3 / 2} e^{-v_{\|}^{2}-\mu B_{0}} \psi_{l} \sum_{n} \psi_{n} \frac{\partial \widehat{\chi}_{n}}{\partial t}=\frac{q_{s}}{T_{s}} \frac{e^{-v_{\|}^{2}}}{\pi^{3 / 2}} \frac{\partial \widehat{\chi}_{l}}{\partial t} .
\end{aligned}
$$



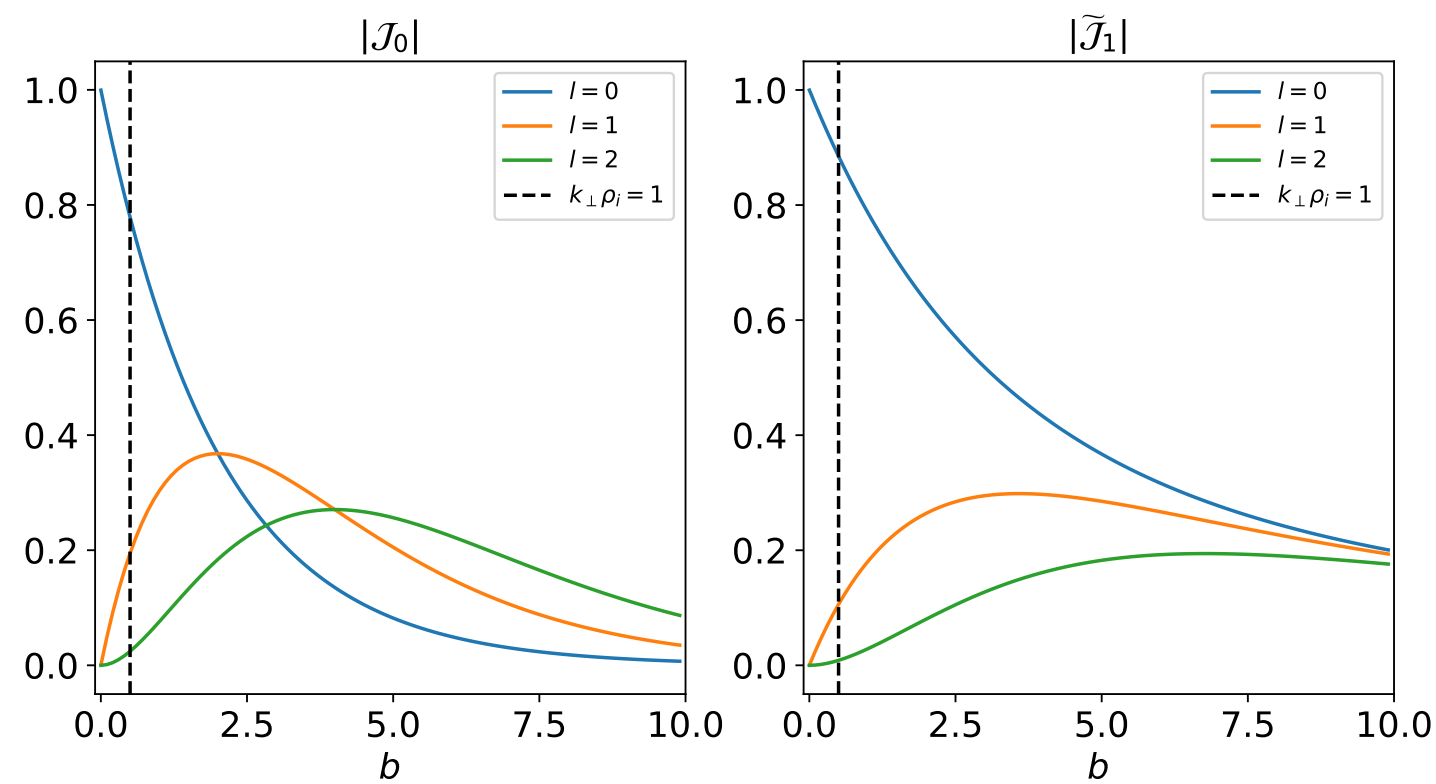

FIG. 1. Absolute value of moments $\mathcal{J}_{0 l}$ and $\tilde{\mathcal{J}}_{1 l}$. With $b \rightarrow 0$, all the higher moments approach zero.

The only complication arises from the nonlinear term, which, as presented in [20, is expressed as a convolution

$$
\int_{0}^{\infty} d \mu B_{0} \frac{1}{B_{0}} \psi_{l}\left\{\sum_{n} \psi_{n} \widehat{\chi}_{n}, \sum_{\kappa} \psi_{\kappa} \widehat{h}_{\kappa}\right\}=\frac{1}{B_{0}} \sum_{\kappa=0}^{\infty} \sum_{n=|\kappa-l|}^{\kappa+l} \alpha_{l \kappa n}\left\{\widehat{\chi}_{n}, \widehat{h}_{\kappa}\right\}
$$

where $\alpha_{l \kappa n}$ is a convolution coefficient computed as

$$
\alpha_{l \kappa n}=\int_{0}^{\infty} d \mu B_{0} \psi_{\kappa} \psi_{n} \psi^{l}=\sum_{j} \frac{(\kappa+l-j) ! 2^{2 j-\kappa-l+n}}{(\kappa-j) !(l-j) !(2 j-\kappa-l+n) !(\kappa+l-n-j) !},
$$

with the summation limits over $j$ being chosen to ensure that the factorials under the sum remain non-negative. Putting all the terms together, gives the GK equation for a Laguerre mode $l$ as

$$
\frac{\partial \widehat{h}_{l}}{\partial t}+\frac{1}{B_{0}} \sum_{\kappa=0}^{\infty} \sum_{n=|\kappa-l|}^{\kappa+l} \alpha_{l \kappa n}\left\{\widehat{\chi}_{n}, \widehat{h}_{\kappa}\right\}+v_{T_{s}} v_{\|} \frac{\partial \widehat{h}_{l}}{\partial z}=\frac{q_{s}}{T_{s}} \frac{e^{-v_{\|}^{2}}}{\pi^{3 / 2}} \frac{\partial \widehat{\chi}_{l}}{\partial t} .
$$

With the GK equation expressed in Laguerre spectral space, we can now take a drift kinetic long wavelength limit, while retaining FLR effects. We do this in the next section and obtain the equations of interests.

\section{DRIFT-KINETIC SYSTEM OF EQUATIONS}

\section{A. Drift-kinetic limit}

In a drift-kinetic approach, the long-wavelength limit $k_{\perp} \rho_{s} \rightarrow 0$ leads to $b_{s} \rightarrow 0$. Dispensing with the species label, for $b \rightarrow 0$ all $l \neq 0$ terms in 20 21) will tend towards zeros (see Figure 1). The only non-zero contributions, for $l=0$, are responsible for retaining FLR effects and have the explicit form,

$$
\begin{aligned}
& \mathcal{J}_{00}=e^{-b / 2}, \\
& \tilde{\mathcal{J}}_{10}=\left[\left(1-e^{-b / 2}\right) \frac{2}{b}\right] .
\end{aligned}
$$


To obtain our equations, which retain the dominant FLR contributions, we set $\mathcal{J}_{0 l}=0$ and $\tilde{\mathcal{J}}_{1 l}=0$ for all $l>0$ in the GK equations expressed in Laguerre form. By doing so, the field equations $(22)-(24)$ now give

$$
\begin{aligned}
\phi(\mathbf{k}, t) & =\pi \sum_{s} q_{s} n_{s} \int_{-\infty}^{+\infty} d v_{\|} \mathcal{J}_{00} \widehat{h}_{s 0} / \sum_{s} \frac{q_{s}^{2} n_{s}}{T_{s}}, \\
A_{\|}(\mathbf{k}, t) & =\frac{\pi \beta}{2 k_{\perp}^{2}} \sum_{s} q_{s} n_{s} v_{T_{s}} \int_{-\infty}^{+\infty} d v_{\|} v_{\|} \mathcal{J}_{00} \widehat{h}_{s 0}, \\
B_{\|}(\mathbf{k}, t) & =-\frac{\pi \beta}{2} \sum_{s} \frac{n_{s} T_{s}}{B_{0}} \int_{-\infty}^{+\infty} d v_{\|} \tilde{J}_{10}\left[\widehat{h}_{s 0}+\widehat{h}_{s 1}\right] .
\end{aligned}
$$

Looking next at the gyrokinetic potential 25$)$, we obtain $\widehat{\chi}_{0}$ and $\widehat{\chi}_{1}$,

$$
\begin{aligned}
& \widehat{\chi}_{0}=\mathcal{J}_{00}\left(\phi(\mathbf{k})-v_{T_{s}} v_{\|} A_{\|}(\mathbf{k})\right)+\frac{T_{s}}{q_{s} B_{0}} B_{\|}(\mathbf{k}) \tilde{\mathcal{J}}_{10}, \\
& \widehat{\chi}_{1}=\frac{T_{s}}{q_{s} B_{0}} B_{\|}(\mathbf{k}) \tilde{\mathcal{J}}_{10}
\end{aligned}
$$

with all other contributions $(l>1)$ being zero.

We now see that while eqs. (34 35) only include the zeroth Laguerre moment of the distribution function $h$, eq. (36) also depends on the first moment. Therefore, one can solve the gyrokinetic system for only the first two moments of the distribution function. The only apparent problem is due to the nonlinear terms, which for $l=0$ equations are given as $\frac{1}{B_{0}} \sum_{\kappa=0}^{\infty} \sum_{n=|\kappa-0|}^{\kappa+0} \alpha_{0 \kappa n}\left\{\widehat{\chi}_{n}, \widehat{h}_{\kappa}\right\}$, and for $l=1$ are found from $\frac{1}{B_{0}} \sum_{\kappa=0}^{\infty} \sum_{n=|\kappa-1|}^{\kappa+1} \alpha_{1 \kappa n}\left\{\widehat{\chi}_{n}, \widehat{h}_{\kappa}\right\}$. However, since for $\widehat{\chi}_{n}$ in the nonlinear terms only $n=0$ and $n=1$ terms are nonzero, and computing the required convolution coefficients $\alpha_{0 \kappa n}$ and $\alpha_{1 \kappa n}$ from (30), we obtain that the nonlinear terms are expressed solely in term of $\widehat{h}_{0}$ and $\widehat{h}_{1}$,

$$
\begin{aligned}
& \frac{\partial \widehat{h}_{0}}{\partial t}+\frac{1}{B_{0}}\left(\left\{\widehat{\chi}_{0}, \widehat{h}_{0}\right\}+\left\{\widehat{\chi}_{1}, \widehat{h}_{1}\right\}\right)+v_{T_{s}} v_{\|} \frac{\partial \widehat{h}_{0}}{\partial z}=\frac{q_{s}}{T_{s}} \frac{e^{-v_{\|}^{2}}}{\pi^{3 / 2}} \frac{\partial \widehat{\chi}_{0}}{\partial t} \\
& \frac{\partial \widehat{h}_{1}}{\partial t}+\frac{1}{B_{0}}\left(\left\{\widehat{\chi}_{0}, \widehat{h}_{1}\right\}+\left\{\widehat{\chi}_{1}, \widehat{h}_{0}\right\}+2\left\{\widehat{\chi}_{1}, \widehat{h}_{1}\right\}\right)+v_{T_{s}} v_{\|} \frac{\partial \widehat{h}_{1}}{\partial z}=\frac{q_{s}}{T_{s}} \frac{e^{-v_{\|}^{2}}}{\pi^{3 / 2}} \frac{\partial \widehat{\chi}_{1}}{\partial t}
\end{aligned}
$$

We see that we obtain a closed set of equations. With $\widehat{h}_{0}=\widehat{h}_{0}\left(\mathbf{R}, v_{\|}\right)$and $\widehat{h}_{1}=\widehat{h}_{1}\left(\mathbf{R}, v_{\|}\right)$obtained explicitly as the largest $\mu$ space scale-structure, our four-dimensional set of equations retain FLR effects. The equations themselves serve to capture electromagnetic plasma turbulence in the drift kinetic limit, while allowing to transition into the sub-gyroradius range of scales captured by GK theory. Our equations account for the linear phase mixing that occurs in $v_{\|}$space due to the particle-field resonance. With an interest in the interplay between spatial turbulence and $v_{\|}$ space mixing, we will perform in the next section a Hermite representation of our equations in the $v_{\|}$direction and a Fourier representation for spatial scales. We first comment on the conservation of free energy and the electrostatic approximation.

\section{B. Free energy conservation}

Following the works of [15, 25], in [29] we took the $\mathbf{R}$-density for the free energy contribution of a species $s$ to be $W_{s}(\mathbf{R}, t)=\pi \int d v_{\|} d \mu B_{0}\left[h_{s}-\frac{q_{s} F_{s}}{T_{s}} \chi\right] \frac{T_{s}}{F_{s}} h_{s}$. Dropping the species label, using for our drift kinetic system $g_{0}=h_{0}-$ $\frac{q_{s}}{T_{s}} e^{-v_{\|}^{2}} \pi^{-3 / 2} \chi_{0}$ and $g_{1}=h_{1}-\frac{q_{s}}{T_{s}} e^{-v_{\|}^{2}} \pi^{-3 / 2} \chi_{1}$, we obtain for in non-dimensional form $W(\mathbf{R}, t)=\pi^{5 / 2} T \int d v_{\|} e^{v_{\|}^{2}}\left[\widehat{h}_{0} \widehat{g}_{0}+\right.$ $\widehat{h}_{1} \widehat{g}_{1}$.

Rewriting eqs. (39) 40 ) as

$$
\begin{aligned}
& \frac{\partial \widehat{g}_{0}}{\partial t}+\frac{1}{B_{0}}\left(\left\{\widehat{\chi}_{0}, \widehat{h}_{0}\right\}+\left\{\widehat{\chi}_{1}, \widehat{h}_{1}\right\}\right)+v_{T_{s}} v_{\|} \frac{\partial \widehat{h}_{0}}{\partial z}=0, \\
& \frac{\partial \widehat{g}_{1}}{\partial t}+\frac{1}{B_{0}}\left(\left\{\widehat{\chi}_{0}, \widehat{h}_{1}\right\}+\left\{\widehat{\chi}_{1}, \widehat{h}_{0}\right\}+2\left\{\widehat{\chi}_{1}, \widehat{h}_{1}\right\}\right)+v_{T_{s}} v_{\|} \frac{\partial \widehat{h}_{1}}{\partial z}=0,
\end{aligned}
$$


multiplying eq. 41) by $\widehat{h}_{0}$ and eq. 42 by $\widehat{h}_{1}$ and summing them we get

$$
\begin{aligned}
& \frac{\partial \widehat{g}_{0}}{\partial t} \widehat{h}_{0}+\frac{\partial \widehat{g}_{1}}{\partial t} \widehat{h}_{1}+ \frac{1}{2} v_{T_{s}} v_{\|}\left[\frac{\partial \widehat{h}_{0}^{2}}{\partial z}+\frac{\partial \widehat{h}_{1}^{2}}{\partial z}\right]+ \\
& \frac{1}{B_{0}}\left[\frac{1}{2}\left\{\widehat{\chi}_{0}, \widehat{h}_{0}^{2}\right\}+\frac{1}{2}\left\{\widehat{\chi}_{0}, \widehat{h}_{1}^{2}\right\}+\left\{\widehat{\chi}_{1}, \widehat{h}_{1}^{2}\right\}+\left\{\widehat{\chi}_{1}, \widehat{h}_{0} \widehat{h}_{1}\right\}\right]=0,
\end{aligned}
$$

where the Poisson bracket and the $z$ derivatives term integrate spatially to zero. We find that $\int d \mathbf{R} \partial W / \partial t \equiv$ $\int d \mathbf{R}\left[h_{0} \partial g_{0} / \partial t+h_{1} \partial g_{1} / \partial t\right]=0$, which shows that the free energy $W$ is conserved globally in the absence of collisions or external energy sources in the system.

\section{Electrostatic case}

The electrostatic limit $(\beta \rightarrow 0)$ represents a special case of interest for our drift-kinetic equations. It can be used to compare with other drift-kinetic models derived solely for electrostatic fluctuations.

With $A_{\|}$and $B_{\|}$fields vanishing, there is no need to keep first Laguerre moment $\left(h_{1}^{m}\right)$ and its associate equations. Therefore, our drift kinetic equations reduce to the one describing the evolution of the zeroth Laguerre moment,

$$
\frac{\partial \widehat{h}_{0}}{\partial t}+\frac{1}{B_{0}}\left\{\widehat{\chi}_{0}, \widehat{h}_{0}\right\}+v_{T_{s}} v_{\|} \frac{\partial \widehat{h}_{0}}{\partial z}=\frac{q}{T} \frac{e^{-v_{\|}^{2}}}{\pi^{3 / 2}} \frac{\partial \widehat{\chi}_{0}}{\partial t}
$$

with

$$
\begin{aligned}
\widehat{\chi}_{0} & =\mathcal{J}_{00} \phi(\mathbf{k}), \\
\phi(\mathbf{k}, t) & =\pi \sum_{s} q_{s} n_{s} \int_{-\infty}^{+\infty} d v_{\|} \mathcal{J}_{00} \widehat{h}_{s 0} / \sum_{s} \frac{q_{s}^{2} n_{s}}{T_{s}} .
\end{aligned}
$$

Going further and selecting an adiabatic species, can lead to a further simplified model as presented in [14] in a Fourier-Hermite spectral form. In the next section we present the Fourier-Hermite spectral form for our system.

\section{DRIFT-KINETIC SYSTEM OF EQUATIONS IN SPECTRAL FORM}

To finish writing our drift kinetic equation is spectral form, we will utilise a Hermite decomposition for $v_{\|}$and a Fourier decomposition in $\mathbf{R}$. We first perform the Hermite representation.

\section{A. Hermite basis projection}

For convenience, we chose the Hermite function basis used by [20. The Hermite functions in question are given as

$$
\begin{aligned}
\xi^{m}\left(v_{\|}\right) & =\frac{(-1)^{m}}{\left(2^{m} m ! \pi^{3}\right)^{1 / 2}} e^{-v_{\|}^{2}} H e\left(v_{\|}\right), \\
\xi_{m}\left(v_{\|}\right) & =\frac{(-1)^{m}}{\left(2^{m} m !\right)^{1 / 2}} H e\left(v_{\|}\right),
\end{aligned}
$$

where $H e\left(v_{\|}\right)=e^{v_{\|}^{2}} \frac{d^{m}}{d v_{\|}^{m}} e^{-v_{\|}^{2}}$ are Hermite polynomials. The Hermite functions obey the orthogonality condition

$$
\pi \int_{-\infty}^{+\infty} d v_{\|} \xi^{m}\left(v_{\|}\right) \xi_{n}\left(v_{\|}\right)=\delta_{n m}
$$

and, among others, the recurrence relation

$$
v_{\|} \xi^{m}\left(v_{\|}\right)=\sqrt{\frac{m}{2}} \xi^{m-1}\left(v_{\|}\right)+\sqrt{\frac{m+1}{2}} \xi^{m+1}\left(v_{\|}\right) .
$$


The decomposition on the Hermite basis is done in the same manner as it was done for the Laguerre basis. Function in Hermite basis can be represented as

$$
f\left(v_{\|}\right)=\sum_{m=0}^{\infty} \xi^{m}\left(v_{\|}\right) f^{m}
$$

with spectral amplitude

$$
f^{m}=\pi \int_{-\infty}^{+\infty} d v_{\|} \xi_{m}\left(v_{\|}\right) f\left(v_{\|}\right)
$$

In Hermite space, by using $\psi_{0}\left(v_{\|}\right)=1$ and the recurrence formula $[50$, the field equations are found to be

$$
\begin{aligned}
\phi(\mathbf{k}, t) & =\pi \sum_{s} q_{s} n_{s} \int_{-\infty}^{+\infty} d v_{\|} \sum_{m=0}^{\infty} \mathcal{J}_{00} \xi^{m}\left(v_{\|}\right) \widehat{h}_{s 0}^{m} / \sum_{s} \frac{q_{s}^{2} n_{s}}{T_{s}} \\
& =\sum_{s} q_{s} n_{s} \mathcal{J}_{00} \widehat{h}_{s 0}^{0} / \sum_{s} \frac{q_{s}^{2} n_{s}}{T_{s}}, \\
A_{\|}(\mathbf{k}, t) & =\frac{\pi \beta}{2 k_{\perp}^{2}} \sum_{s} q_{s} n_{s} v_{T_{s}} \int_{-\infty}^{+\infty} d v_{\|} v_{\|} \mathcal{J}_{00} \sum_{m=0}^{\infty} \xi^{m}\left(v_{\|}\right) \widehat{h}_{s 0}^{m} \\
& =\frac{\pi \beta}{2 k_{\perp}^{2}} \sum_{s} q_{s} n_{s} v_{T_{s}} \int_{-\infty}^{+\infty} d v_{\|} \mathcal{J}_{00} \sum_{m=0}^{\infty}\left(\sqrt{\frac{m}{2}} \xi^{m-1}\left(v_{\|}\right)+\sqrt{\frac{m+1}{2}} \xi^{m+1}\left(v_{\|}\right)\right) \widehat{h}_{s 0}^{m} \\
& =\frac{\beta}{2 k_{\perp}^{2}} \sum_{s} q_{s} n_{s} v_{T_{s}} \mathcal{J}_{00} \sqrt{\frac{1}{2}} \widehat{h}_{s 0}^{1}, \\
B_{\|}(\mathbf{k}, t) & =-\frac{\beta}{2} \sum_{s} \frac{n_{s} T_{s}}{B_{0}} \tilde{\mathcal{J}}_{10}\left(\widehat{h}_{s 0}^{0}+\widehat{h}_{s 1}^{0}\right)
\end{aligned}
$$

where for $B_{\|}$the procedure is the same as for (53). To help with the representation of the GK equation in Hermite space, as the $A_{\|}$contribution contains a $v_{\|}$factor, and the $B_{\|}$contribution is the same in (37) and (38), we represent $\widehat{\chi}_{0}$ and $\widehat{\chi}_{1}$ as

$$
\begin{aligned}
& \widehat{\chi}_{0}=\widehat{\chi}^{\phi}+\widehat{\chi}^{B}+v_{\|} \widehat{\chi}^{A}, \\
& \widehat{\chi}_{1}=\widehat{\chi}^{B},
\end{aligned}
$$

with

$$
\begin{aligned}
\widehat{\chi}^{\phi}(\mathbf{k}, t) & =\mathcal{J}_{00} \phi(\mathbf{k}, t), \\
\widehat{\chi}^{B}(\mathbf{k}, t) & =\frac{T}{q B_{0}} \tilde{\mathcal{J}}_{10} B_{\|}(\mathbf{k}, t), \\
\widehat{\chi}^{A}(\mathbf{k}, t) & =-v_{T_{s}} \mathcal{J}_{00} A_{\|}(\mathbf{k}, t) .
\end{aligned}
$$

The projection of the GK equation on the Hermite basis is done in straightforward manner. One has to use (51) for $h_{0}$ and $h_{1}$, the recurrence formula $\left[50\right.$ for terms that contain $v_{\|}$, and the integrate over $\pi \int_{-\infty}^{+\infty} d v_{\|} \xi_{m}$. As an example we look at the nonlinear term $\frac{1}{B_{0}}\left\{\chi_{0}, h_{0}\right\}$, which becomes

$$
\begin{aligned}
\frac{\pi}{B_{0}} \int_{-\infty}^{+\infty} d v_{\|} \xi_{m} & \left\{\widehat{\chi}^{\phi}+\widehat{\chi}^{B}+v_{\|} \widehat{\chi}^{A}, \sum_{n} \xi^{n} \widehat{h}_{0}^{n}\right\}=\frac{1}{B_{0}}\left\{\widehat{\chi}^{\phi}, \widehat{h}_{0}^{m}\right\}+\frac{1}{B_{0}}\left\{\widehat{\chi}^{B}, \widehat{h}_{0}^{m}\right\} \\
& +\frac{1}{B_{0}} \sqrt{\frac{m+1}{2}}\left\{\widehat{\chi}^{A}, \widehat{h}_{0}^{m+1}\right\}+\frac{1}{B_{0}} \sqrt{\frac{m}{2}}\left\{\widehat{\chi}^{A}, \widehat{h}_{0}^{m-1}\right\} .
\end{aligned}
$$


The GK equation expressed in Hermite space have the form

$$
\begin{aligned}
& \frac{\partial \widehat{h}_{0}^{m}}{\partial t}+\frac{1}{B_{0}}\left[\left\{\widehat{\chi}^{\phi}+\widehat{\chi}^{B}, \widehat{h}_{0}^{m}\right\}+\sqrt{\frac{m+1}{2}}\left\{\widehat{\chi}^{A}, \widehat{h}_{0}^{m+1}\right\}+\sqrt{\frac{m}{2}}\left\{\widehat{\chi}^{A}, \widehat{h}_{0}^{m-1}\right\}+\left\{\widehat{\chi}^{B}, \widehat{h}_{1}^{m}\right\}\right] \\
& +v_{T_{s}} \frac{\partial}{\partial z}\left[\sqrt{\frac{m+1}{2}} \widehat{h}_{0}^{m+1}+\sqrt{\frac{m}{2}} \widehat{h}_{0}^{m-1}\right]=\frac{q}{T} \frac{\partial}{\partial t}\left[\left(\widehat{\chi}^{\phi}+\widehat{\chi}^{B}\right) \delta_{m 0}+\sqrt{\frac{1}{2}} \widehat{\chi}^{A} \delta_{m, 1}\right] \\
& \frac{\partial h_{1}^{m}}{\partial t}+\frac{1}{B_{0}}\left[\left\{\widehat{\chi}^{\phi}+\widehat{\chi}^{B}, \widehat{h}_{1}^{m}\right\}+\sqrt{\frac{m+1}{2}}\left\{\widehat{\chi}^{A}, \widehat{h}_{1}^{m+1}\right\}+\sqrt{\frac{m}{2}}\left\{\widehat{\chi}^{A}, \widehat{h}_{1}^{m-1}\right\}+\left\{\widehat{\chi}^{B}, \widehat{h}_{0}^{m}\right\}\right. \\
& \left.+2\left\{\widehat{\chi}^{B}, \widehat{h}_{1}^{m}\right\}\right]+v_{T_{s}} \frac{\partial}{\partial z}\left[\sqrt{\frac{m+1}{2}} \widehat{h}_{1}^{m+1}+\sqrt{\frac{m}{2}} \widehat{h}_{1}^{m-1}\right]=\frac{q}{T} \frac{\partial \widehat{\chi}^{B}}{\partial t} \delta_{m, 0}
\end{aligned}
$$

Expressing these equation in Fourier space is trivial and we will simply present them in the next section.

\section{B. Drift-kinetic system in spectral space}

To keep notations as simple as possible, we write the Fourier space representation for the Poisson bracket between two functions $f$ and $g$ as

$$
\{f, g\}(\mathbf{k})=\frac{1}{2} \sum_{\mathbf{p}+\mathbf{q}=\mathbf{k}}\left(q_{x} p_{y}-q_{y} p_{x}\right)[\widehat{f}(\mathbf{q}) \widehat{g}(\mathbf{p})-\widehat{f}(\mathbf{p}) \widehat{g}(\mathbf{q})] .
$$

Dispensing with the $\hat{\ldots}$ designation for (Fourier-Hermite-Laguerre space) modes to further simplify notations, the drift-kinetic equations in spectral form have the form

$$
\begin{aligned}
\frac{\partial g_{0}^{m}}{\partial t} & +\frac{1}{B_{0}}\left[\left\{\chi^{\phi}+\chi^{B}, h_{0}^{m}\right\}+\sqrt{\frac{m+1}{2}}\left\{\chi^{A}, h_{0}^{m+1}\right\}+\sqrt{\frac{m}{2}}\left\{\chi^{A}, h_{0}^{m-1}\right\}\right. \\
& \left.+\left\{\chi^{B}, h_{1}^{m}\right\}\right]+i k_{z} v_{T_{s}}\left[\sqrt{\frac{m+1}{2}} h_{0}^{m+1}+\sqrt{\frac{m}{2}} h_{0}^{m-1}\right]=0 \\
\frac{\partial g_{1}^{m}}{\partial t} & +\frac{1}{B_{0}}\left[\left\{\chi^{\phi}+\chi^{B}, h_{1}^{m}\right\}+\sqrt{\frac{m+1}{2}}\left\{\chi^{A}, h_{1}^{m+1}\right\}+\sqrt{\frac{m}{2}}\left\{\chi^{A}, h_{1}^{m-1}\right\}\right. \\
& \left.+\left\{\chi^{B}, h_{0}^{m}\right\}+2\left\{\chi^{B}, h_{1}^{m}\right\}\right]+i k_{z} v_{T_{s}}\left[\sqrt{\frac{m+1}{2}} h_{1}^{m+1}+\sqrt{\frac{m}{2}} h_{1}^{m-1}\right]=0
\end{aligned}
$$

where it is convenient to use the modified gyrokinetic distribution functions,

$$
\begin{aligned}
& g_{0}^{m}=h_{0}^{m}-\frac{q}{T}\left(\chi^{\phi}+\chi^{B}\right) \delta_{m 0}-\frac{q}{T} \sqrt{\frac{1}{2}} \chi^{A} \delta_{m 1} \\
& g_{1}^{m}=h_{1}^{m}-\frac{q}{T} \chi^{B} \delta_{m 0}
\end{aligned}
$$

with the GK potential contributions given as

$$
\begin{aligned}
\chi^{\phi}(\mathbf{k}) & =\mathcal{J}_{00} \phi(\mathbf{k}), \\
\chi^{B}(\mathbf{k}) & =\frac{T}{q B_{0}} \tilde{\mathcal{J}}_{10} B_{\|}(\mathbf{k}), \\
\chi^{A}(\mathbf{k}) & =-v_{T_{s}} \mathcal{J}_{00} A_{\|}(\mathbf{k}) .
\end{aligned}
$$


for fields

$$
\begin{aligned}
\phi(\mathbf{k}, t) & =\sum_{s} q_{s} n_{s} \mathcal{J}_{00} h_{s 0}^{0} / \sum_{s} \frac{q_{s}^{2} n_{s}}{T_{s}}, \\
A_{\|}(\mathbf{k}, t) & =\frac{\beta}{2 k_{\perp}^{2}} \sum_{s} q_{s} n_{s} v_{T_{s}} \mathcal{J}_{00} \sqrt{\frac{1}{2}} h_{s 0}^{1}, \\
B_{\|}(\mathbf{k}, t) & =-\frac{\beta}{2} \sum_{s} \frac{n_{s} T_{s}}{B_{0}} \tilde{\mathcal{J}}_{10}\left[h_{s 0}^{0}+h_{s 1}^{0}\right],
\end{aligned}
$$

and Bessel function contributions that account for FLR effects,

$$
\begin{aligned}
\mathcal{J}_{00} & =e^{-b / 2} \\
\tilde{\mathcal{J}}_{10} & =\left[\left(1-e^{-b / 2}\right) \frac{2}{b}\right] .
\end{aligned}
$$

These are the equations that will be solved numerically in a pseudo-spectral form or in a triad reduced model for the nonlinear terms. We have not include a collision operator in our presentation. One can chose to use the one presented in [20, or simply use a Laplacian or hyper-Laplacian expressed locally in $m$ and $k_{\perp}$ space as means to provide a sink of free energy at small scales. However, in the collisionless regimes, the dissipation of free energy from a truncated range of scales is due to an anomalous dissipation generated by the flux of free energy to the unresolved scales [11. With this discussion being lengthly and requiring numerical results to support it, we choose not to delve in the presentation of any dissipative mechanism.

\section{CONCLUSIONS}

The goal of this article was to derive a set of four-dimensional drift kinetic equations that conserve free energy, and are capable of describing electromagnetic turbulence in a magnetised plasma at the transition between fluid and GK regimes $\left(k_{\perp} \rho_{i} \approx 1\right.$ range of scales). Starting from the GK equations, we have employed a Laguerre decomposition of the distribution functions in the perpendicular velocity direction and have retained only the first two Laguerre moments that source the electromagnetic fluctuations. FLR effects are kept via the dominant contributions of the Bessel functions, expanded in a Laguerre polynomial basis, allowing for the transition into the GK range of scales to be possible.

The Laguerre-Hermite representation has been used in 20 to present a spectral approach for electrostatic GK in toroidal magnetic geometry. Here, not only that we expanded this to electromagnetic fluctuations, we have have effectively integrated out the $\mu$ dependence to reduce the dimensions of the kinetic system at $k_{\perp} \rho_{i}<1$. In addition to the three-dimensions in positions space, we retained the parallel velocity dependence, which we captured via a Hermite representation. Employing this system, but without any other physics based assumptions for the plasma species, will allow us to investigate how fluid effects transition into the kinetic range in the astrophysical context.

Currently, there are a large number of models describing the astrophysical plasma in the large span of scales natural for such system [1, 25, 42. In general, they all can be combined into three big groups: (i) kinetic models that take into account all the kinetic effects, (ii) MHD models that treat plasma as a fluid of charged particles, (iii) and kineticreduced MHD (KRMHD) models [19, 21] that assume isothermal (fluid) electrons, which are applicable for scales smaller or approximately to that of the ion gyroradius. The latter models provide the computational effectiveness, and are able to capture some important kinetic effects. However, since most of those models utilise additional physical assumptions to eliminate one of the species, some of the kinetic effects can fall out of the scope of these models, or they can be captured in a very slim way. Therefore, our choice was to use the route highlighted by [5] and derive a more comprehensive drift-kinetic model, in order to track down kinetic effects arising at the border of transition from fluid to (gyro)kinetic turbulence. Our approach is better suited to the analysis of the interplay between spatial and velocity space mixing for electromagnetic plasma turbulence, as no a-priori assumption on the fluid nature of a species is made. This will be pursued numerically via pseudo-spectral solvers and low dimensional models. 


\section{ACKNOWLEDGEMENTS}

BT would like to thank Gabriel Plunk and David Hatch for discussions on GK dynamics and reduced models.

[1] Adkins, T. \& Schekochinin, A. A. 2018 A solvable model of vlasov-kinetic plasma turbulence in fourier-hermite phase space. Journal of Plasma Physics 84 (1), 905840107.

[2] Alexandrova, O., Carbone, V., Veltri, P. \& Sorriso-Valvo, L. 2008 Small-scale energy cascade of the solar wind turbulence. The Astrophysical Journal 674, 1153.

[3] Alexandrova, O., Saur, J., Lacombe, C., Mangeney, A., Mitchell, J., Schwartz, S. J. \& Robert, P. 2009 Universality of solar-wind turbulent spectrum from mhd to electron scales. Phys. Rev. Lett. 103, 165003.

[4] Bhattacharjee, A., NG, C. S. \& Spangler, S. R. 1998 Weakly compressible magnetohydrodynamic turbulence in the solar wind and the interstellar medium. The Astrophysical Journal 494, 409.

[5] Brizard, A. 1992 Nonlinear gyrofluid description of turbulent magnetized plasmas. Physics of Fluids B: Plasma Physics $4(5), 1213-1228$.

[6] Cerri, S. S., Kunz, M. W. \& Califano, F. 2018 Dual phase-space cascades in 3d hybrid-vlasov-maxwell turbulence. The Astrophysical Journal Letters 856, L13.

[7] Chandran, B., Quataert, E., Howes, G. G., Hollweg, J. V. \& Dorland, W. 2009 The turbulent heating rate in strong magnetohydrodynamic turbulence with nonzero cross helicity. The Astrophysical Journal 701, 652.

[8] Dorland, W. \& Hammett, G. W. 1993 Gyrofluid turbulence models with kinetic effects. Physics of Fluids B: Plasma Physics 5 (3), 812-835.

[9] Duan, D., Bowen, T. A., Chen, C. H. K., Mallet, A., He, J., Bale, S. D., Vech, D., Kasper, J. C., Pulupa, M., Bonnell, J. W., Case, A. W., de Wit, T. D., Goetz, K., Harvey, P. R., Korreck, K. E., Larson, D., Livi, R., MacDowall, R. J., Malaspina, D. M., Stevens, M. \& Whittlesey, P. 2020 The radial dependence of proton-scale magnetic spectral break in slow solar wind during PSP encounter 2. The Astrophysical Journal Supplement Series 246 (2), 55.

[10] Duan, D., He, J., Bowen, T. A., Woodham, L. D., Wang, T., Chen, C. H. K., Mallet, A. \& Bale, S. D. 2021 Anisotropy of solar wind turbulence in the inner heliosphere at kinetic scales: PSP observations. The Astrophysical Journal Letters 915 (1), L8.

[11] Eyink, G. L. 2018 Cascades and dissipative anomalies in nearly collisionless plasma turbulence. Phys. Rev. X 8, 041020.

[12] Goldreich, P. \& Sridhar, S. 1995 Toward a theory of interstellar turbulence. 2: Strong alfvenic turbulence. The Astrophysical Journal 438, 763.

[13] Grošelu, D., Chen, C. H. K., Mallet, A., Samtaney, R., Schneider, K. \& Jenko, F. 2019 Kinetic Turbulence in Astrophysical Plasmas: Waves and/or Structures? Physical Review X 9 (3), 031037.

[14] Hatch, D. R., Jenko, F., Bratanov, V. \& Navarro, A. B. 2014 Phase space scales of free energy dissipation in gradient-driven gyrokinetic turbulence. Journal of Plasma Physics 80 (4), 531-551.

[15] Howes, G. G., Cowley, S. C., Dorland, W., Hammett, G. W., Quataert, E. \& Schekochinin, A. A. 2006 Astrophysical gyrokinetics: Basic equations and linear theory. The Astrophysical Journal 651, 590.

[16] Howes, G. G., Dorland, W., Cowley, S. C., Hammett, G. W., Quataert, E., Schekochinin, A. A. \& Tatsuno, T. 2008 Kinetic simulations of magnetized turbulence in astrophysical plasmas. Phys. Rev. Lett. 100, 65004.

[17] Howes, G. G., Tenbarge, J. M., Dorland, W., Quataert, E., Schekochinin, A. A., Numata, R. \& Tatsuno, T. 2011 Gyrokinetic simulations of solar wind turbulence from ion to electron scales. Phys. Rev. Lett. 107, 35004.

[18] Kawazura, Y., Barnes, M. \& Schekochinin, A. A. 2019 Thermal disequilibration of ions and electrons by collisionless plasma turbulence. Proceedings of the National Academy of Sciences 116 (3), 771-776.

[19] Kunz, M., Abel, I., Klein, K. \& Schekochinin, A. 2018 Astrophysical gyrokinetics: turbulence in pressure-anisotropic plasmas at ion scales and beyond. Journal of Plasma Physics 84 (2), 715840201.

[20] Mandell, N., Dorland, W. \& Landreman, M. 2018 Laguerre-hermite pseudo-spectral velocity formulation of gyrokinetics. Journal of Plasma Physics 84 (1), 905840108.

[21] Meyrand, R., Kanekar, A., Dorland, W. \& Schekochinin, A. A. 2019 Fluidization of collisionless plasma turbulence. Proceedings of the National Academy of Sciences 116 (4), 1185-1194.

[22] Meyrand, R., Squire, J., Schekochinin, A. \& Dorland, W. 2021 On the violation of the zeroth law of turbulence in space plasmas. Journal of Plasma Physics 87 (3), 535870301.

[23] Navarro, A. B., Teaca, B., Told, D., Groselu, D., Crandall, P. \& Jenko, F. 2016 Structure of plasma heating in gyrokinetic alfvénic turbulence. Phys. Rev. Lett. 117 (24), 245101.

[24] Schekochinin, A. A., Cowley, S. C., Dorland, W., Hammett, G. W., Howes, G. G., Plunk, G. G., Quataert, E. \& Tatsuno, T. 2008 Gyrokinetic turbulence: a nonlinear route to dissipation through phase space. Plasma Phys. Control. Fusion 50, 4024.

[25] Schekochinin, A. A., Cowley, S. C., Dorland, W., Hammett, G. W., Howes, G. G., Quataert, E. \& Tatsuno, T. 2009 Astrophysical Gyrokinetics: Kinetic and Fluid Turbulent Cascades in Magnetized Weakly Collisional Plasmas. The Astrophysical Journal Supplement Series 182 (1), 310-377.

[26] Schekochinin, A. A., Parker, J. T., Highcock, E. G., Dellar, P. J., Dorland, W. \& Hammett, G. W. 2016 
Phase mixing versus nonlinear advection in drift-kinetic plasma turbulence. Journal of Plasma Physics 82 (2), 905820212.

[27] Servidio, S., Valentini, F., Califano, F. \& Veltri, P. 2012 Local kinetic effects in two-dimensional plasma turbulence. Phys. Rev. Lett. 108, 045001.

[28] Tatsuno, T., Dorland, W., Schekochinin, A. A., Plunk, G. G., Barnes, M., Cowley, S. C. \& Howes, G. G. 2009 Nonlinear phase mixing and phase-space cascade of entropy in gyrokinetic plasma turbulence. Phys. Rev. Lett. 103, 15003.

[29] Teaca, B., Gorbunov, E. A., Told, D., Bañón Navarro, A. \& Jenko, F. 2021 Sub-grid-scale effects in magnetised plasma turbulence. Journal of Plasma Physics 87 (2), 905870209.

[30] Teaca, B., Jenko, F. \& Told, D. 2017 Gyrokinetic turbulence: between idealized estimates and a detailed analysis of nonlinear energy transfers. New J. Phys. 19, 045001.

[31] Teaca, B., Navarro, A. B. \& Jenko, F. 2014 The energetic coupling of scales in gyrokinetic plasma turbulence. Phys. Plasmas 21, 072308.

[32] Teaca, B., Navarro, A. B., Jenko, F., Brunner, S. \& Villard, L. 2012 Locality and universality in gyrokinetic turbulence. Phys. Rev. Lett. 109, 235003.

[33] Teaca, B., Navarro, A. B., Told, D., Görler, T., Plunk, G., Hatch, D. R. \& Jenko, F. 2019 A look at phase space intermittency in magnetized plasma turbulence. The Astrophysical Journal 886, 65.

[34] Tenbarge, J. M. \& Howes, G. G. 2013 Current sheets and collisionless damping in kinetic plasma turbulence. The Astrophysical Journal Letters 771, L27.

[35] Told, D., Jenko, F., TenBarge, J. M., Howes, G. G. \& Hammett, G. W. 2015 Multiscale nature of the dissipation range in gyrokinetic simulations of alfvénic turbulence. Phys. Rev. Lett. 115, 025003.

[36] Vech, D., Kasper, J. C., Klein, K. G., Huang, J., Stevens, M. L., Chen, C. H. K., Case, A. W., Korreck, K., Bale, S. D., Bowen, T. A., Whittlesey, P. L., Livi, R., Larson, D. E., Malaspina, D., Pulupa, M., Bonnell, J., Harvey, P., Goetz, K., De Wit, T. D. \& MacDowall, R. 2020 Kinetic-scale spectral features of cross helicity and residual energy in the inner heliosphere. The Astrophysical Journal Supplement Series 246 (2), 52.

[37] Wan, M., Matthaeus, W. H., Karimabadi, H., Roytershteyn, V., Shay, M., Wu, P., Daughton, W., Loring, B. \& Chapman, S. C. 2012 Intermittent dissipation at kinetic scales in collisionless plasma turbulence. Phys. Rev. Lett. 109, 195001.

[38] Weidl, M. S., Jenko, F., Teaca, B. \& Schlickeiser, R. 2015 Cosmic-ray pitch-angle scattering in imbalanced mhd turbulence simulations. The Astrophysical Journal 811, 8.

[39] Zhao, L.-L., Zank, G. P., Adhikari, L., Nakanotani, M., Telloni, D. \& Carbone, F. 2020 Spectral features in field-aligned solar wind turbulence from parker solar probe observations. The Astrophysical Journal 898 (2), 113.

[40] Zhdankin, V., Boldyrev, S., Mason, J. \& Perez, J. C. 2012 Magnetic discontinuities in magnetohydrodynamic turbulence and in the solar wind. Phys. Rev. Lett. 108, 175004.

[41] Zhou, Y., Matthaeus, W. H. \& Dmitruk, P. 2004 Colloquium: Magnetohydrodynamic turbulence and time scales in astrophysical and space plasmas. Rev. Mod. Phys. 76, 1015.

[42] Zocco, A. \& Schekochinin, A. A. 2011 Reduced fluid-kinetic equations for low-frequency dynamics, magnetic reconnection, and electron heating in low-beta plasmas. Physics of Plasmas 18 (10), 102309. 